\title{
Performance Comparison of Different Routing Protocols for Traffic Monitoring Application
}

\author{
Anand Mehrotra \\ PSIT College of Engineering \\ Sr Lecturer
}

\author{
Arti Saxena \\ PSIT College of Engineering \\ Asst Prof. (HOD)
}

\author{
Manoj Tolani \\ PSIT College of Engineering \\ Asst Professor
}

\begin{abstract}
Now days the majority of research has been done using simulation only. Simulation as well as Practical Models has their importance. For implementation using simulator knowledge of routing protocol is required, further ad hoc routing protocols are different from traditional routing protocols. In this paper OPNET 14.5 is used for comparing performance of different routing protocols AODV, OSLR, DSR, GRP, and TORA in traffic monitoring application. The model is drawn for campus of $22.5 \mathrm{~m} \mathrm{X} 22.5 \mathrm{~m}$ and by changing protocols in each scenario and increasing number of nodes the performance analysis is done.
\end{abstract}

\section{General Terms}

\section{Keywords}

AODV (Ad-hoc On-demand Distance Vector routing protocol), OSLR (Optimized Link State routing protocol), DSR (Dynamic Source Routing Protocol), GRP (Geographic routing protocols), TORA (Temporally Ordered Routing Algorithm)

\section{INTRODUCTION}

Now a day's wireless networks are used to allow users to communicate with each other. Ad hoc wireless network must be capable to self-organize and self-configure due to the fact that the mobile structure is changing all the time. The mobile host must be capable of broadcasting the messages and should be in mode for accepting messages that it receives.

The key issues associated with the routing protocols for ad hoc wireless network are: It must be capable to handle a very large number of hosts with limited resources, such as bandwidth and energy. It has to deal with host mobility, since hosts can appear and disappear at various locations. In this particular paper immobile nodes are considered but the results can be extended to the mobile nodes. In Ad hoc network all nodes act as routers. Another important issue is keeping the routing table small because its increase will affect the control packets sent in the network and which will affect large link overheads.

This paper will compare the five ad hoc routing protocols: AODV, OLSR, DSR, GRP, TORA,[7],[8] the reminding part of this paper is organized as follows. Sec. 2 will give information about various routing protocols, Sec. 3 discusses about the traffic control application, sections 4 discusses about the theoretical effect of increasing nodes Actual comparison will be done in Sec. 5 with all simulation results attached ,Sec 6 discusses about the future work possibilities ,Sec. 7 will conclude this paper.

\section{ROUTING PROTOCOLS}

Routing protocols are of two types: Proactive (for e.g. OLSR, WRP etc) and Reactive (for e.g. AODV, DSR, TORA).[4]

\subsection{Proactive routing (Table- Driven) protocols}

In this protocol, all the nodes continuously search for routing information within a network, Every node maintains one or more tables representing the entire topology of the network.

\subsection{Reactive Routing (On-Demand) protocols}

Routing information is collected only when it is needed, and route determination depends on sending route queries throughout the network.

\section{ROUTING PROTOCOLS CONSIDERED IN TNIS PAPER}

\subsection{AODV (Ad-hoc On-demand Distance Vector routing protocol)}

It is a routing protocol which is used for dynamic wireless networks. In this the information of topology is transmitted on-demand by nodes. To find a route to a particular destination node. In this source node sends a RREQ to its immediate neighbors. On receiving the request neighbor node checks if it has route to the destination, then it replies back with a RREP. If the neighboring node has no route to the destination then RREQ is flooded to other nodes until destination node is achieved on recognizing the destination node chain of RREP messages is sent back.

\subsection{OSLR (Optimized Link State routing protocol)}

OLSR is a table-driven pro-active protocol. It uses the linkstate scheme in an optimized manner to diffuse topology information so as to preserve bandwidth. The optimization is based on a technique called Multi Point Relaying.

\subsection{DSR (Dynamic Source Routing protocol)}

It is a reactive protocol, in which route is determined when apacket needs to be send. The node forwards the route request to other nodes and builds the route from the responses it receives. DSR is completely self-configuring and it does not needs any existing network infrastructure or administration.

\subsection{GRP (Geographic routing protocols)}

In Geographic Routing Protocol data is routed using geographical position rather than their network address, in GRP each node determine its own geographical address. 


\subsection{TORA (Temporally Ordered Routing}

\section{Algorithm)}

It is based on the concept of link reversal; it is an adaptive loop-free distributed routing algorithm. It provides multiple routes for any desired source/destination. In TORA near the topological change localization of control messages to a very small set of nodes is done. For achieving this information about adjacent node is maintained. The protocol performs three basic functions: Route creation, Route maintenance, Route erasure.[6]

\section{TRAFFIC MONITORING}

\section{APPLICATION}

This model is designed for monitoring traffic. In this model 15 scenarios is created with different protocols,

In scenario 1 AODV protocol is used

In scenario 2 OLSR protocol is used
In scenario 4 GRP protocol is used

In scenario 5 TORA protocol is used

Scenario 6 to 15 number of nodes is increased to study their effect on throughput

In scenario 6,11 AODV protocol is used and number of nodes are increased.

In scenario 7,12 OLSR protocol is used and number of nodes are increased.

In scenario 8,13 DSR protocol is used and number of nodes are increased.

In scenario 9,14 GRP protocol is used and number of nodes are increased.

In scenario 10,15 TORA protocol is used and number of nodes are increased.

In scenario 3 DSR protocol is used

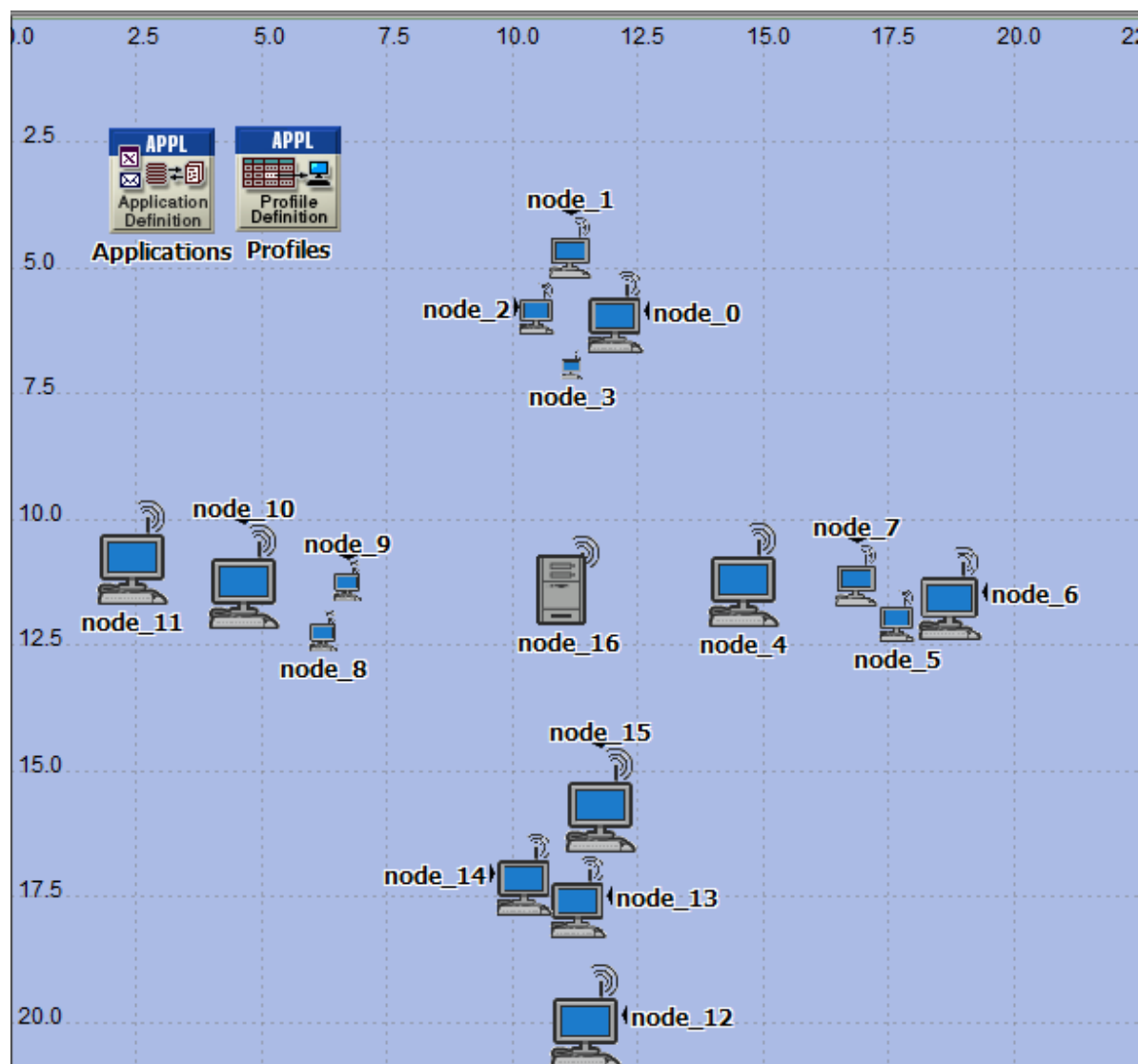

Fig 1: Scenario 1 to 5 


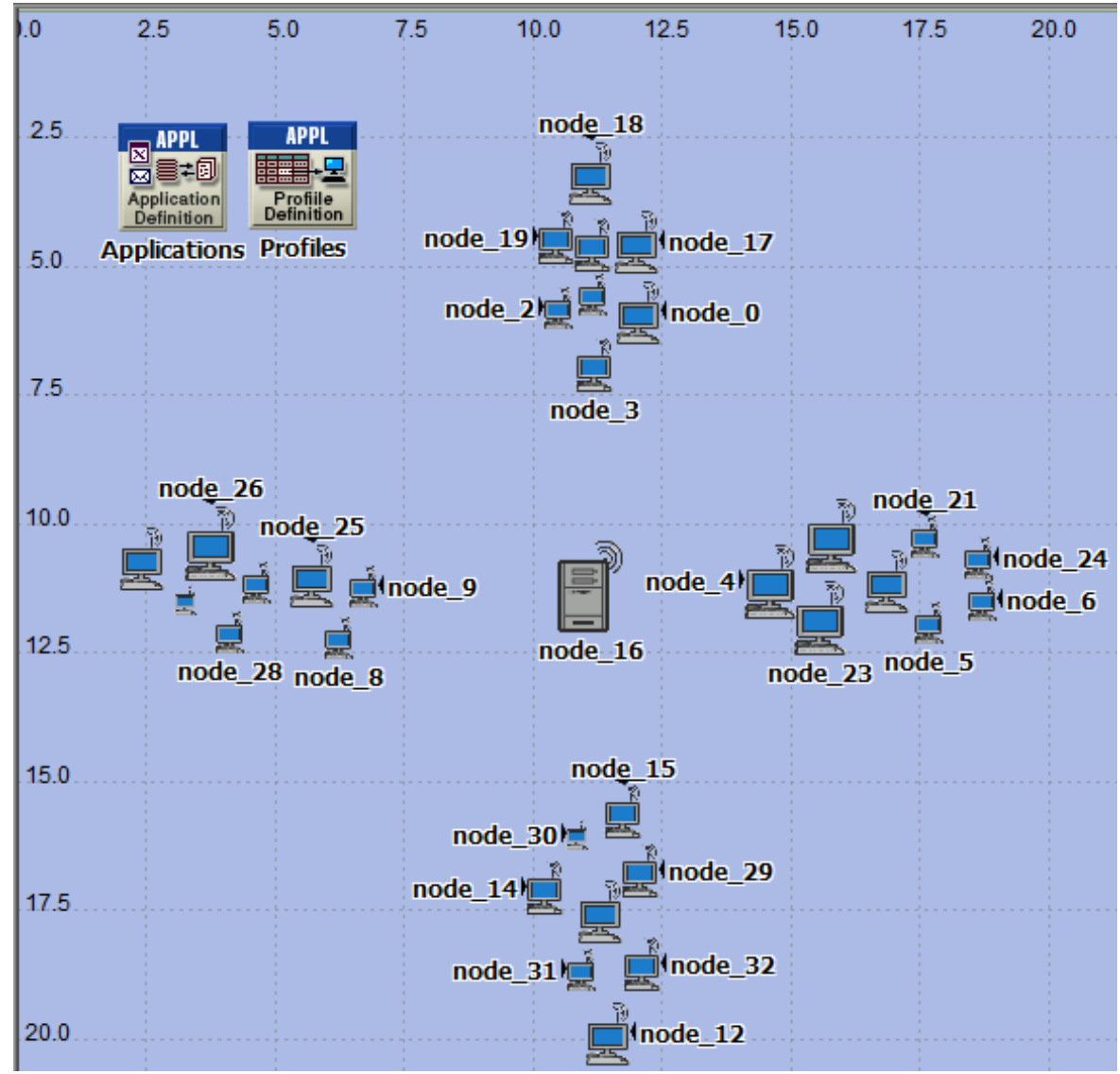

Fig 2: Scenario 6 to 10

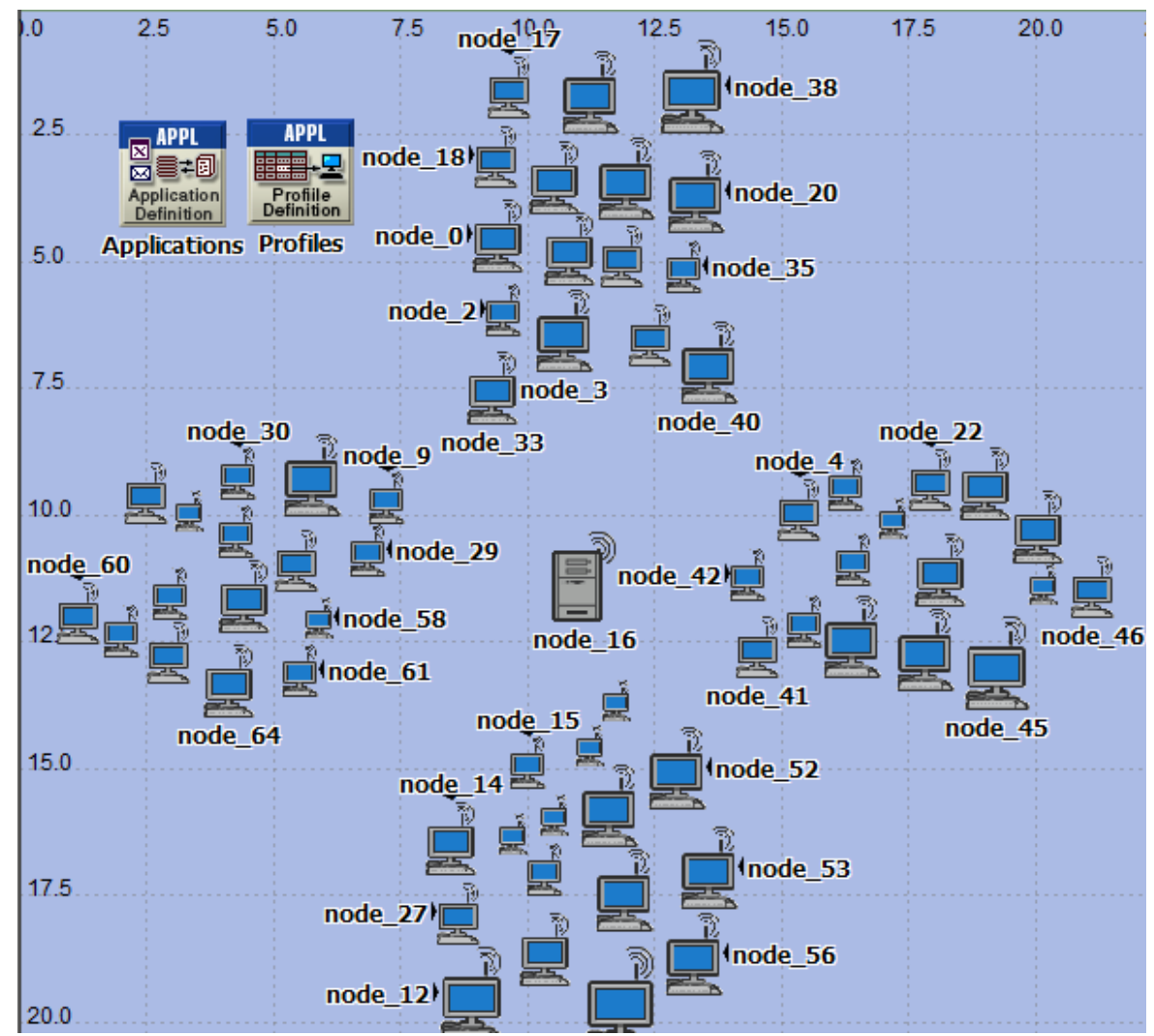

Fig 3: Scenario 11 to 15 


\section{EFFECT OF INCEASING NODES ON THROUGHPUT}

In Ad hoc network every node act as router and the performance of any wireless protocol depends on the duration of interconnections between any two nodes transferring data as well on the duration of interconnections between nodes of a data path containing nodes.

The number of the nodes affects the number of average connected paths, which also affect the performance of the routing algorithm. With very sparsely populated network the number of possible connection between any two nodes is very less and hence the performance is poor. It is expected that if the node density is increased the throughput of the network shall increase, but beyond a certain level if density is increased the performance degrades.[3][5]

\section{SIMULATION RESULTS}

The model is drawn for campus of $22.5 \mathrm{~m} \mathrm{X} 22.5 \mathrm{~m}$ and by changing protocols in each scenario and increasing number of nodes the performance analysis is done.[9][10]

In Fig 4 Throughput of all the protocols are compared. On comparing OLSR and GRP has approximately same throughput.

In Fig 5 Time averaged simulation result of throughput for all protocols is done.

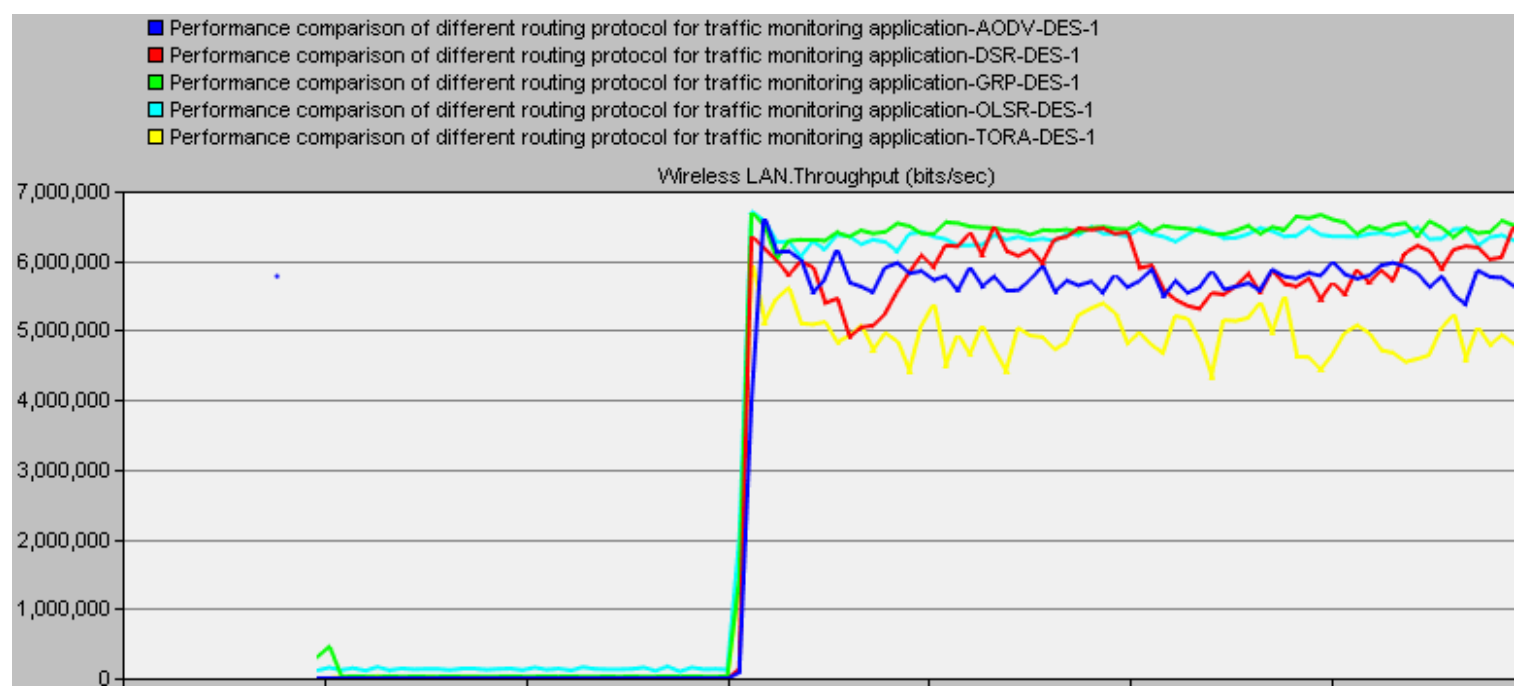

Fig 4: Comparison of Exact simulation result of all the protocols 
- Performance comparison of different routing protocol for traffic monitoring application-AODV-DES-1

$\square$ Performance comparison of different routing protocol for traffic monitoring application-DSR-DES-1

$\square$ Performance comparison of different routing protocol for traffic monitoring application-GRP-DES-1

口 Performance comparison of different routing protocol for traffic monitoring application-OLSR-DES-1

$\square$ Performance comparison of different routing protocol for traffic monitoring application-TORA-DES-1

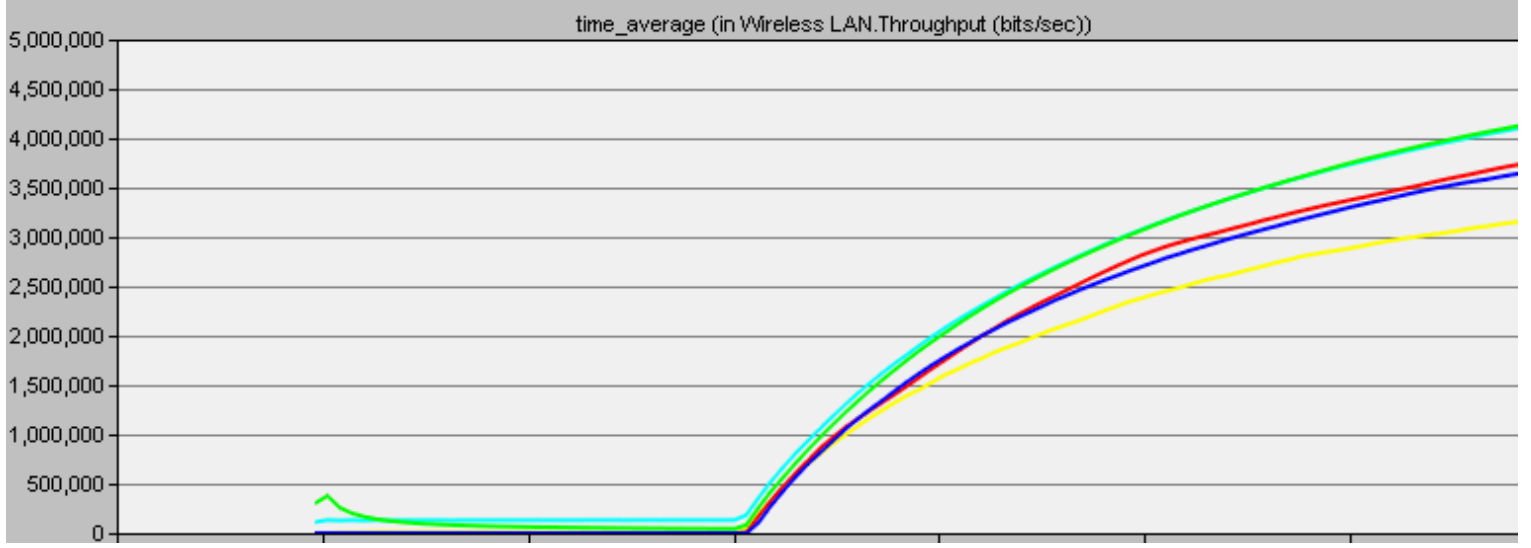

Fig 5: Comparison of Time averaged simulation result of throughput for all protocols

Performance comparison of different routing protocol for traffic monitoring application-GRP-DES-1

$\square$ Performance comparison of different routing protocol for traffic monitoring application-OLSR-DES-1

time_average (in Wireless LAN.Delay (sec))

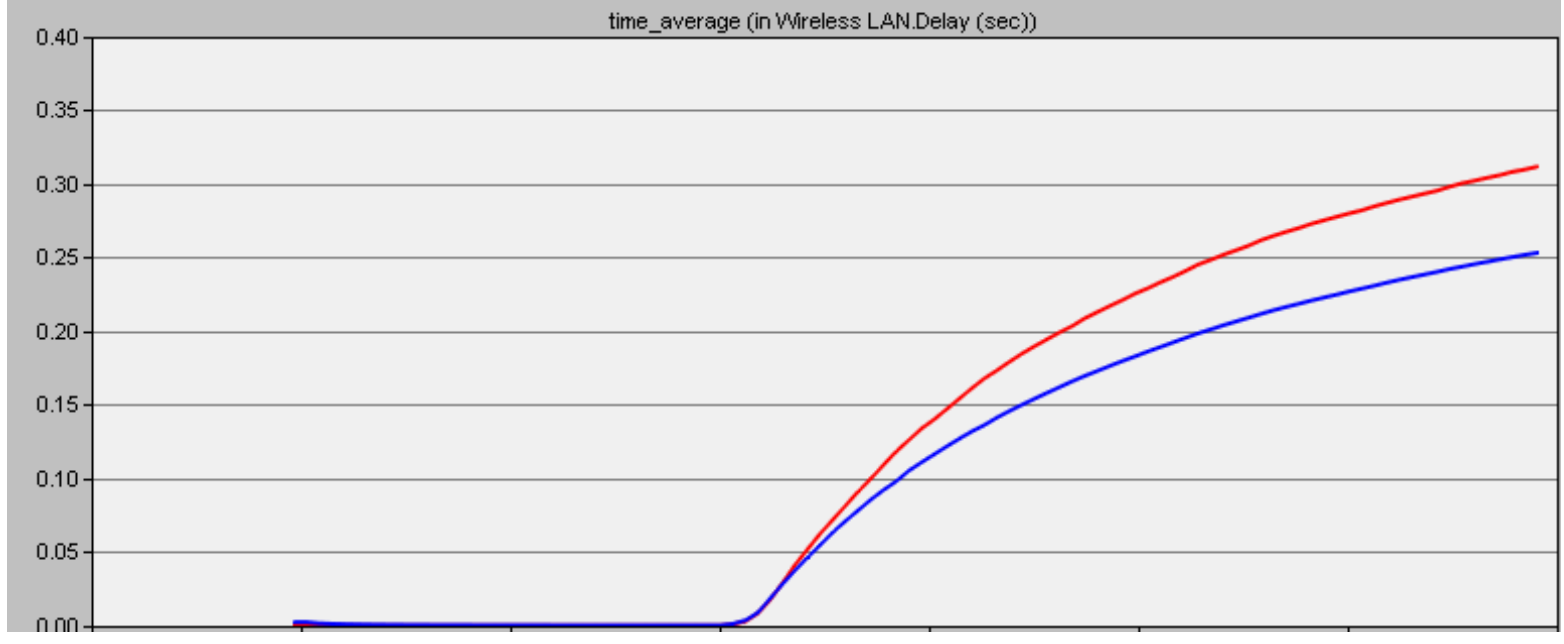

Fig 6: Delay comparison of OLSR and GRP

Performance comparison of different routing protocol for traffic monitoring application-OLSR 8 NODES-DES-1

$\square$ Performance comparison of different routing protocol for traffic monitoring application-OLSR 16 NODES-DES-1

$\square$ Performance comparison of different routing protocol for traffic monitoring application-OLSR-DES-1

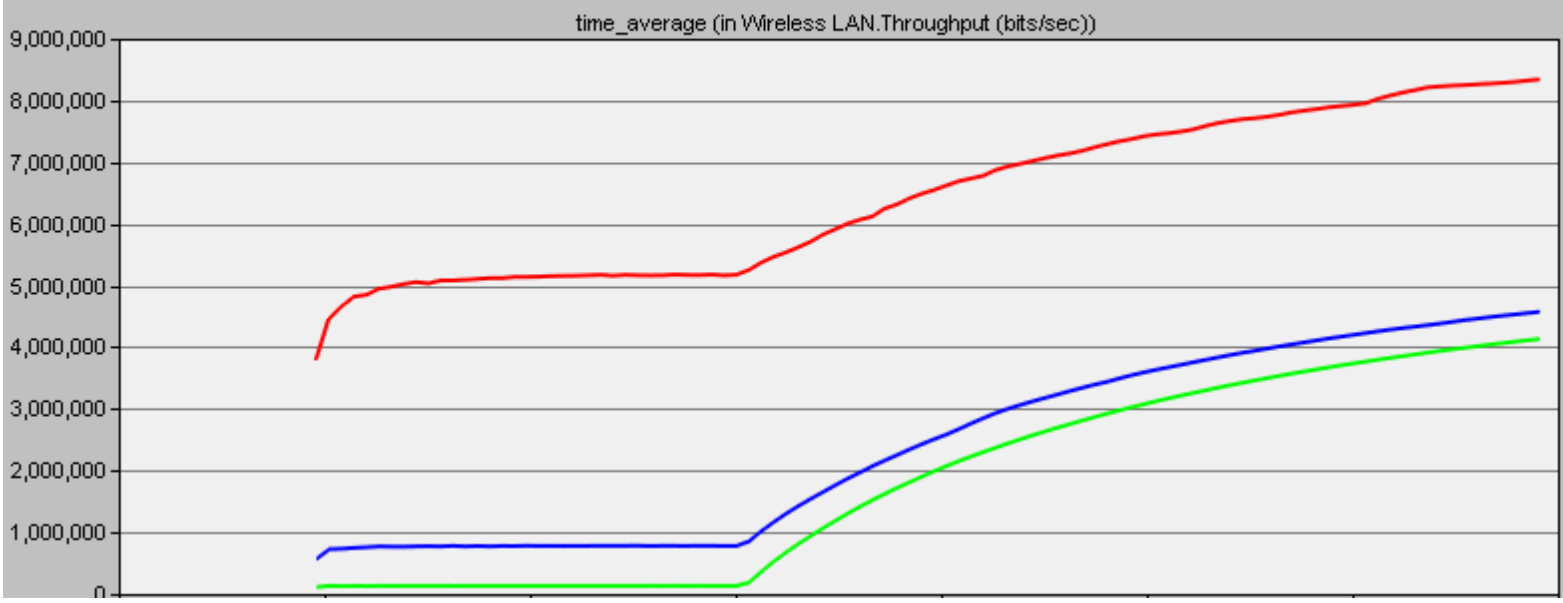

Fig 7: Comparison of time averaged throughput on increasing number of nodes for OLSR. 


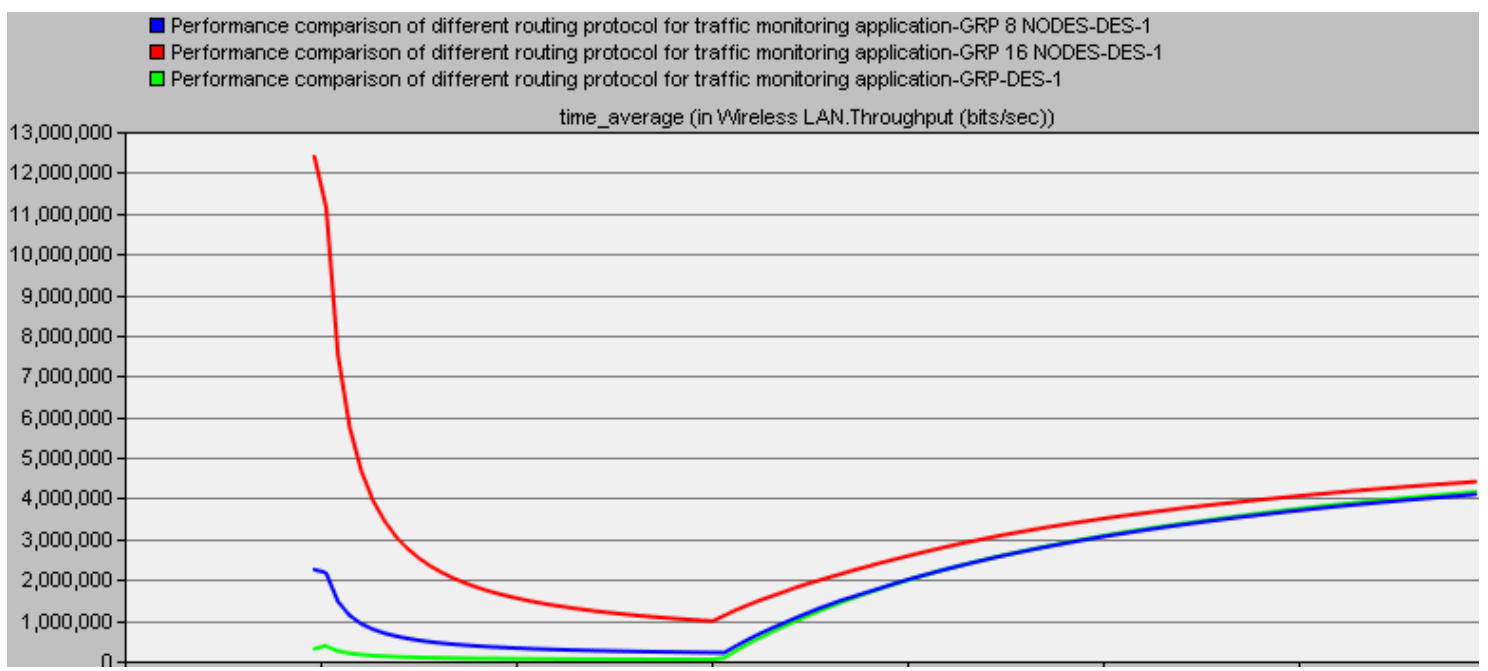

Fig 8: Comparison of time averaged throughput on increasing number of nodes for GRP.

\section{CONCLUSIONS}

In this paper various routing protocols are compared and it is observed that GRP and OLSR are the best while considering throughput calculation (by taking time average among all other protocols). Among GRP and OLSR, GRP is the best since by studying the delay graph of these protocols GRP has less delay. On increasing the number of nodes in each scenario and using different protocols it is found that throughput increases on increasing number of nodes.

\section{FUTURE WORK}

This paper is written by considering a campus of $22.5 \mathrm{~m} \mathrm{X}$ $22.5 \mathrm{~m}$ for traffic control application for immobile nodes but the results can be extended for mobile nodes moreover exact geographical data can be entered and the comparison can be done for specific location in the world for practical analysis..

\section{REFERENCES}

[1] Patrick Sondi, DhavyGantsou and Sylvain Lecomte, "Mobile Ad Hoc Network-Based Monitoring Of Battlefields Or Rescue Operations In Urban Scenarios",UKSim Fourth European Modelling Symposium on Computer Modelling and Simulation, pages pp 408-413,IEEE 2010.

[2] Zahian Ismail, Rosilah Hassan, "Effects Of Packet Size On AODV Routing Protocol Implementation In Homogeneous and Heterogeneous MANET", 2011 Third International Conference on Computational Intelligence, Modelling\& Simulation, pages pp 351-356, IEEE 2011.

[3] MostafaFazeli, HasanVaziri, "Assessment Of Throughput Performance Under OPNET Modeler Simulation Tools In Mobile Ad Hoc Networks (MANET's)", 2011 Third International Conference on Computational Intelligence, Communication Systems and Networks,pages pp 328-331, IEEE 2011.

[4] AshishShrestha, FiratTekiner, "On MANET Routing Protocols for Mobility and Scalability" 2009 International Conference on Parallel and Distributed
Computing, Applications and Technologies, pages pp 451-456, IEEE 2009.

[5] R.K.Nadesh , D.Sumathy, M. B. BenjulaAnbu Malar,"Performance Analysis Of MANET (WLAN) Using Different Routing Protocols In Multi Service Environments-An Quantitative Study", Int. J. Advanced Networking and Applications Volume: 03, Issue: 02, Pages:1076-1079 (2011)

[6] Anuj K. Gupta, Member, IACSIT, Dr. Harsh Sadawarti, Dr. Anil K. Verma, "Performance Analysis Of AODV, DSR \& TORA Routing Protocols", IACSIT International Journal of Engineering and Technology, Vol.2, No.2,pages pp 226-231 April 2010.

[7] Al-Maashri, A. and Ould-Khaoua, M. (2006) "Performance Analysis Of MANET Routing Protocols in the presence of Self-Similar Traffic", Proceedings of the 31st IEEE Conference on Local Computer Networks,2006, 14-16 November 2006, pages pp. 801807, Tampa, Florida, USA.

[8] Yi Wang, Hairong Chen, Xinyu Yang and Deyun Zhang, "Cluster Based Location-Aware Routing Protocol For Large Scale Heterogeneous Manet", Second International Multisymposium on Computer and Computational Science, Computer Society pages pp 366-373, IEEE (2007).

[9] EmadAboelela, "Computer Networks A System Approach, Edition-3, Networks Simulation Experiment Mannual",University of Massachusetts Dartmouth, 2003.

[10] TanviMalik,Gaurav Mittal, Monika Aggarwal "Simulation Based Performance Analysis of Ad-Hoc Routing Protocols in Various Moving Trajectories", Department of ECE, B.G.IET, Sangrur, India, Vol 1, Issue 7, 373-375, IJCSET August2011 